\title{
Interaction between superconducting vortices and a Bloch wall in ferrite garnet films
}

\author{
J. I. Vestgården, D. V. Shantsev, A. A. F. Olsen, Y. M. Galperin, V. V. Yurchenko, P. E. Goa and T. H. Johansen \\ Department of Physics and Center for Materials Science and Nanotechnology, \\ University of Oslo, P. O. Box 1048 Blindern, 0316 Oslo, Norway
}

\begin{abstract}
A theoretical model for how Bloch walls occurring in in-plane magnetized ferrite garnet films can serve as efficient magnetic micro-manipulators is presented. As example, the walls' interaction with Abrikosov vortices in a superconductor in close contact with a garnet film is analyzed within the London approximation. The model explains how vortices are attracted to such walls, and excellent quantitative agreement is obtained for the resulting peaked flux profile determined experimentally in $\mathrm{NbSe}_{2}$ using high-resolution magneto-optical imaging of vortices. In particular, this model, when generalized to include charged magnetic walls, explains the counter-intuitive attraction observed between vortices and a Bloch wall of opposite polarity.
\end{abstract}

Microscopic magnetic potentials offer an efficient and often indispensable way to manipulate various tiny objects, e.g., to trap and guide Bose-Einstein condensates and degenerate Fermi gases of ultracold atoms $1,2,3$ or to stretch and twist DNA strands attached to paramagnetic beads 4.5 . How precisely one can position the trapped object and how large forces can be applied are determined by the steepness of the magnetic potential produced by the manipulating device. Typically, an assembly of microfabricated wires or coils can generate fields with gradients up to $10^{2}-10^{3} \mathrm{~T} / \mathrm{m}^{1,2,6,7}$. Recently, magnetic manipulators were created using ferrimagnetic films, where domain walls create locally even stronger field gradients. In particular, ferrite-garnet films (FGFs) were used to trap ultracold neutral atoms ${ }^{8}$, assemble and guide colloidal particles $\frac{9.10}{}$, and manipulate vortices in superconductors ${ }^{11}$. Using in-plane magnetized FGFs, where domains are separated by Bloch walls, has two strong advantages: (i) it is easy to move the domain wall and thereby tune the magnetic potential, and (ii) one can simultaneously observe the motion of the manipulated objects. This "see what you do" ability stems from the large Faraday effect in the FGFs, which today are extensively used as magneto-optical imaging (MOI) sensors 12 . In an optical polarizing microscope configuration the FGF allows direct visualization of both the stray field from the manipulated magnetic objects and the Faraday rotation in the wall itself.

In this work we present a theoretical model for how Bloch walls can function as magnetic manipulators, using as example their interaction with a lattice of Abrikosov vortices in a type-II superconductor. The configuration considered is that of a FGF located near, but a finite distance from the surface of a flat superconductor. It is shown that the model, generalized to include charged walls, explains how the vortices are attracted to such walls, and predicts quantitatively the non-uniform flux density distribution we find experimentally using MOI.

Fig. 1 shows an image of the magnetic field distribution near two linear Bloch wall segments, which themselves appear dark in the image. The superconductor is a single crystal of $\mathrm{NbSe}_{2} \frac{13}{}$ cooled to $T=4 \mathrm{~K}$ in a $0.3 \mathrm{mT}$ perpendicular magnetic field, which transformed

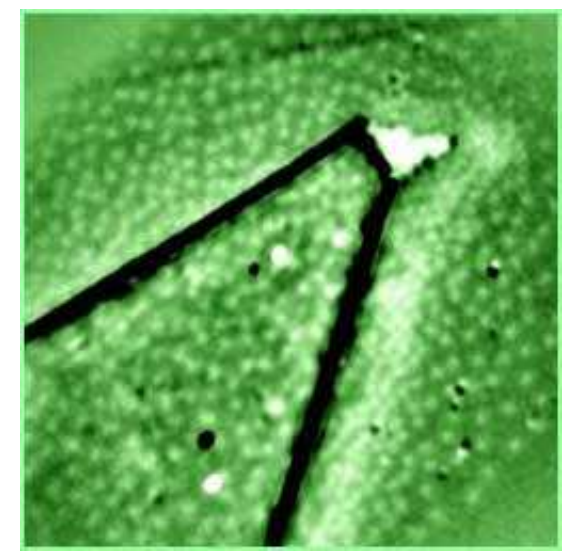

FIG. 1: Magneto-optical image of the vortex distribution near a Bloch wall in a Bi-substituted lutetium iron garnet film placed on top of a superconducting $\mathrm{NbSe}_{2}$ crystal with transition temperature of $7.2 \mathrm{~K}$. The slightly uncrossed analyzer and polarizer setting used here implies that the dark wall and the bright vortices have opposite field polarities. Image dimensions are $70 \times 70 \mu \mathrm{m}^{2}$.

into quantized vortices when entering the superconducting state. Each vortex is here seen as a bright dot. Evidently, the magnetic wall has a considerable attraction on the vortices, since their number density increases near the wall. This observation forms the experimental basis for the theoretical modeling presented below. Interestingly, a most surprising feature visible from Fig. 1 makes the problem even more challenging. There is opposite contrast between the dark wall and the bright vortices, which means they are of opposite magnetic polarity. In this case one would expect from the models presented previously in the literature $\frac{14,15,16,17}{1}$ that a Bloch wall should repel the vortices. As will be shown, by accounting for additional magnetic charges due to misalignment between the wall direction and the in-plane magnetization vector within the domains, the sign of the interaction can become inverted.

The two Bloch wall segments seen in Fig. 1 1 are actually part of a larger zigzag pattern. Extended zigzag domain walls are commonly present in FGFs with strong inplane anisotropy $\frac{18}{2}$. An example is shown in Fig. 2 (top), 

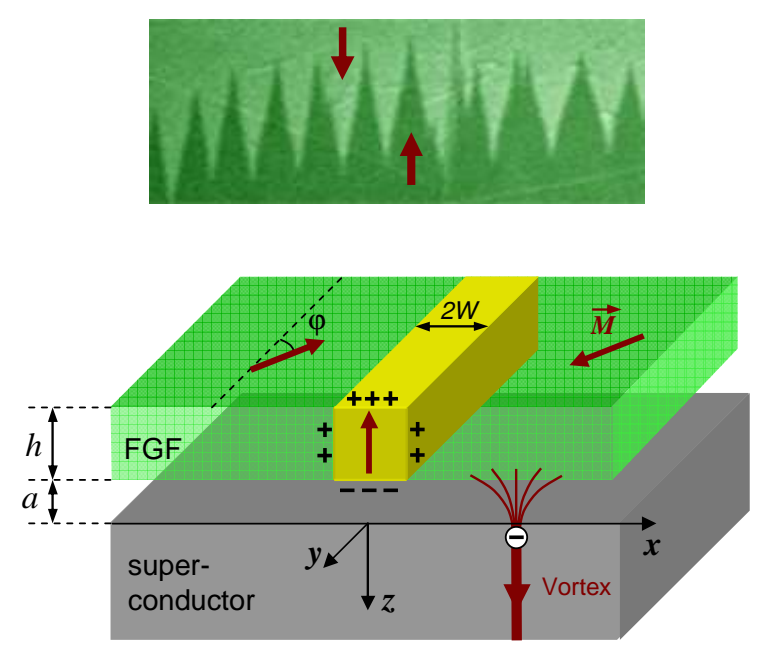

FIG. 2: Top: MO image showing a zigzag Bloch wall in a FGF separating two domains with antiparallel in-plane magnetization. Bottom: Sketch of an in-plane magnetized FGF with a Bloch wall placed above a superconductor. The magnetic charges along the vertical sides of the wall can lead to a net attraction between a wall and vortices, as seen in Fig. 1 .

where the zigzag line separates two domains with antiparallel magnetizations that meet head-on. By folding into a zigzag pattern the domain boundary reduces the density of magnetic charges at the wall, which helps to minimize the energy $\underline{\underline{19}}$. To describe the interaction between one segment of such a zigzag wall and a superconducting vortex, we introduce the model illustrated in Fig. 2 (bottom). The superconductor occupies the halfspace, $z>0$, and the wall is directed along the $y$ axis, which forms an angle $\varphi$ with the magnetization direction of the two domains. Experimentally we detect only tiny changes of the wall width, $2 W$, during cooling through $T_{c}$, in agreement with 14 . Thus, in calculations the wall is approximated as a fixed, uniform, out-of-plane magnetization $M_{z}(|x|<W)=-M_{s}$. Inside the domains there is an in-plane magnetization with a component normal to the wall given by $M_{x}(|x|>W)=-M_{s}(x /|x|) \sin \varphi$, where $M_{s}$ is the saturation magnetization. For $\varphi=0^{\circ}$ the present model reduces to the non-charged wall case. The $M_{y}$ component is omitted in the analysis since the wall is assumed to be infinitely long.

Stray fields from the wall induce shielding currents in the superconductor, which we determine using the London theory. The equations valid inside and outside the superconductor then read

$$
\begin{aligned}
\lambda^{-2} A-\nabla^{2} A & =0 & , z \geq 0 \\
-\nabla^{2} A & =\mu_{0}(\nabla \times \mathbf{M})_{y} & , z \leq 0
\end{aligned}
$$

where $\lambda$ is the London penetration depth and the vector potential is $\mathbf{A}=A \hat{y}$. The shielding currents flow in the $y$ direction and their density equals $J_{y}=-A / \mu_{0} \lambda^{2}$. A vortex present in the superconductor then feels two forces. First, the direct force from the FGF, which can be cal-

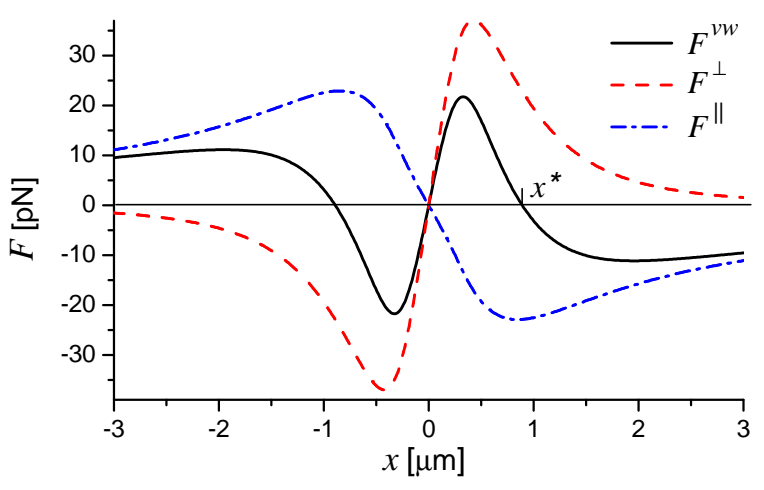

FIG. 3: The calculated forces on the vortex from the Bloch wall: $F^{\perp}$ is repulsive and $F^{\|}$is attractive. Their sum $F^{v w}$ changes sign at $x^{*} \approx 1 \mu \mathrm{m}$. The parameters used here are: $\sin \varphi=0.34,2 W=0.6 \mu \mathrm{m}, h=0.8 \mu \mathrm{m}, a=140 \mathrm{~nm}$, $\lambda=200 \mathrm{~nm}$, and $M_{s}=50 \mathrm{kA} / \mathrm{m}$.

culated from the free energy term, $\mu_{0} \int \mathbf{M} \cdot \mathbf{H}_{v} d V$, where $H_{v}$ is the stray field from the vortex. Second, the Lorentz force from the shielding currents in the superconductor, $\mathbf{F}_{L}=\mathbf{J}(\mathbf{r}) \times \boldsymbol{\Phi}_{0}$ integrated over the length of the vortex. $\Phi_{0}$ is the magnetic flux quantum, and we will simplify the treatment by assuming the vortex to be straight and aligned with the $z$ axis. Interestingly, the two forces turn out to have exactly the same magnitude and direction, as was noted also in Ref. 15 where a similar configuration was analyzed.

It is convenient to express the total force on the vortex in the $x$ direction as

$$
F^{v w}=F^{\perp}+F^{\|}
$$

where $F^{\perp}$ and $F^{\|}$are the contributions from the perpendicular and in-plane components of $\mathbf{M}$, respectively. Their Fourier transforms are obtained as

$$
\begin{aligned}
& F_{k}^{\perp}=-4 i \frac{M_{s} \Phi_{0}}{\lambda^{2}} \frac{1-e^{-|k| h}}{|k| \tau(\tau+|k|)} e^{-|k| a} \sin W k, \\
& F_{k}^{\|}=4 i \frac{M_{s} \Phi_{0}}{\lambda^{2}} \frac{1-e^{-|k| h}}{k \tau(\tau+|k|)} e^{-|k| a} \cos W k \sin \varphi
\end{aligned}
$$

where $\tau=\sqrt{\lambda^{-2}+k^{2}}, a$ is the gap between superconductor and FGF, and $h$ is the FGF thickness. For the configuration illustrated in Fig. 2 the force $F^{\|}$is always attractive, whereas $F^{\perp}$ is repulsive. This qualitative result can be easily understood by considering the interaction between the magnetic charges involved. The stray field from a vortex is closely approximated by that of a magnetic monopole located at $z \sim \lambda$ and with strength $-2 \Phi_{0} \stackrel{20}{ }$. Thus, the vortex is attracted to the positive charges along the vertical sides of the wall and repelled by the perpendicular dipole charges. The charge representation also yields the correct magnitude of forces given by Eqs. (3) and (4) in the limit $\lambda \rightarrow 0$. The superconductor then perfectly screens the magnetic field created by $\mathbf{M}$ and its presence should be accounted for by adding the corresponding mirror charges. 


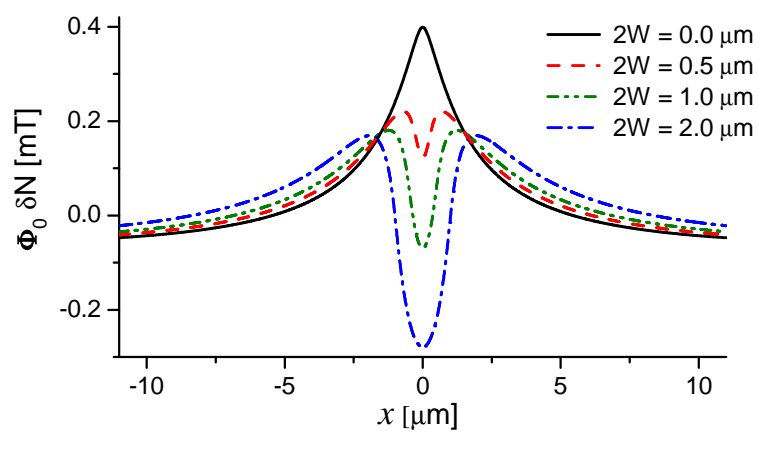

FIG. 4: The excess vortex density $\delta N(x)$ near the Bloch wall for various wall width $2 W$ calculated using $L=160 \mu \mathrm{m}$ and the other parameter values as indicated in the caption of Fig. 3

From the inverse transform of (3) and (4), we obtain the spatial variation of the two force contributions, which are plotted in Fig. 3 together with the total force on the vortex. At sufficiently large $x$ the magnitude of $F^{\|}$is larger than that of $F^{\perp}$, which is expected since the monopole-monopole interaction should dominate at long distances. However, at short distances $F^{\perp}$ becomes dominant, and the total force changes sign at some distance $x^{*}$. For our set of parameters, the repulsive region $|x|<x^{*}$ is very small, less than $1 \mu \mathrm{m}$. This is why the repulsive region under the wall is not visible in the image of Fig. 1. This also explains why we observe the counterintuitive attraction between the Bloch wall and the vortices of opposite polarity. A related phenomenon when ferromagnetic domain wall stimulates superconductivity due to its stray fields was considered in Ref. 21.

We consider next an initial state with a uniform distribution of vortices in the superconductor, and a subsequent introduction of a Bloch wall. This results in a perturbation of the vortex density, $\delta N(x)$, which creates an additional force acting on every vortex. In the equilibrium, the additional force everywhere balances the force from the Bloch wall, i.e.,

$$
F^{v w}(x)=\int F^{v v}\left(x-x^{\prime}, y^{\prime}\right) \delta N\left(x^{\prime}\right) d x^{\prime} d y^{\prime},
$$

where $F^{v v}$ is the $x$ component of the repulsive vortexvortex interaction, and the vortex matter is considered as a continuum. This equation represents a perfect shielding of the domain wall by the vortex matter. In Fourier space, the perturbed vortex density becomes $\delta N_{k}=F_{k}^{v w} / F_{k}^{v v}$. The vortex-vortex interaction can be obtained from the currents around a flux line in a half-space, first calculated by Pearl22, and gives

$$
F_{k}^{v v}=\frac{\Phi_{0}^{2}}{\mu_{0} \lambda^{2}} \frac{i k}{|k| \tau^{2}}\left(|k| L+\frac{1}{\lambda^{2}} \frac{1}{\tau} \frac{1}{|k|+\tau}\right)
$$

where $L$ is the flux line length. The term proportional to $L$ is the conventional (Abrikosov) bulk contribution, while the other term is the surface contribution.
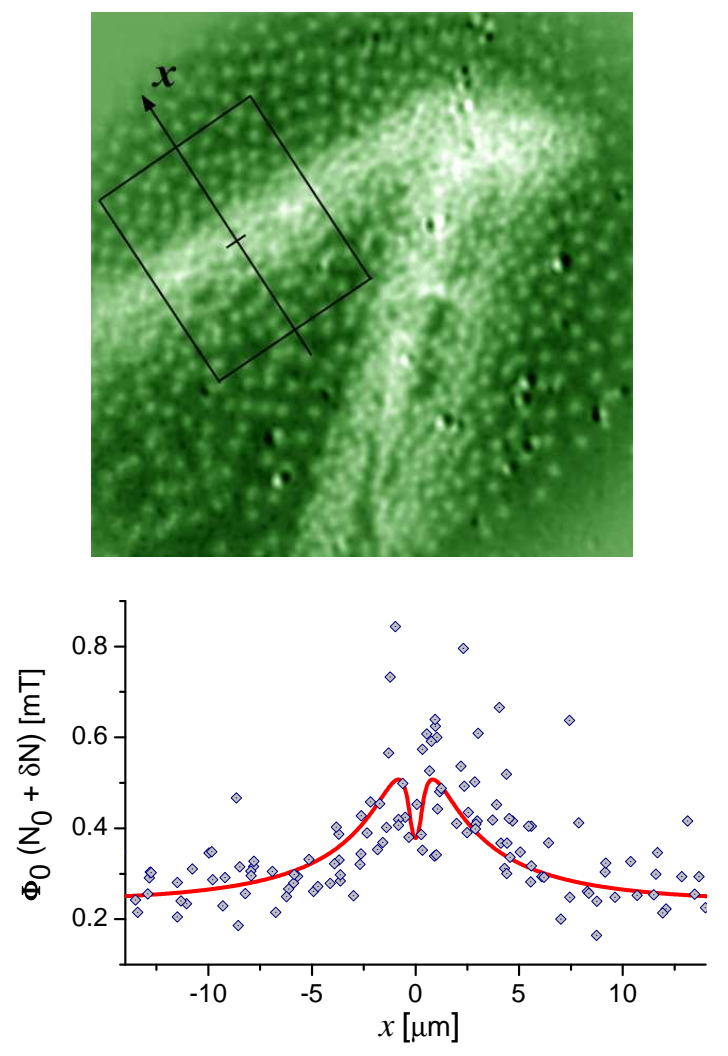

FIG. 5: Top: Distribution of vortices formed in the presence of a Bloch wall. The image was taken after the wall, seen in Fig. 1, was removed by an in-plane field of the order of a few $\mu \mathrm{T}$ applied perpendicular to the indicated $\mathrm{x}$-axis. Bottom: Vortex density obtained from the image (each symbol represents one vortex) together with the theoretical curve calculated for $L=160 \mu \mathrm{m}, \Phi_{0} N_{0}=0.3 \mathrm{mT}$ and remaining parameters as listed in the caption of Fig. 3 .

The resulting perturbation $\delta N(x)$ of the vortex density induced by a Bloch wall is shown in Fig. 4. Its profile is strongly dependent on the wall width $2 W$. In the small $W$ limit the total force on a vortex is everywhere attractive, hence the vortex density increases monotonously as one approaches the wall. For increasing $W$ the density profile develops a minimum below the center of the wall, and the resulting $\delta N(x)$ becomes non-monotonous with a minimum at the center and maxima near the two wall edges. For sufficiently large $W$ the vortices right below the wall are expelled creating a narrow depleted area.

The theoretical vortex density profile $\delta N(x)$ can be compared to our MOI observations of the vortex distribution near the Bloch wall. The FGF had a thickness $h=$ $0.8 \mu \mathrm{m}$, and saturation magnetization $M_{s}=50 \mathrm{kA} / \mathrm{m}$. The film, with no additional layers (contrary to standard MOI indicators), was placed directly on top of the $0.3 \mathrm{~mm}$ thick $\mathrm{NbSe}_{2}$ crystal with a gap of $a=140 \mathrm{~nm}$, as determined from the optical interference pattern 23 . This gap equals one quarter of a wavelength,and gives optimal transmission.

The vortices were formed slightly below $T_{c}$ where flux 
pinning is negligible. Thus, one expects that the vortex positions seen in Fig. 11 represent a frozen picture of an arrangement where the vortices adjust only to balance the interaction with the wall. To obtain a better view, we made use of the fact that further cooling to $4 \mathrm{~K}$ increased the vortex pinning considerably, and removed the Bloch wall without creating noticeable change in the vortex positions, see and Fig. 5 (top). From this image, the positions of all the vortices inside the marked rectangular area were identified, and the Wigner-Seitz cell of each vortex was determined using standard triangulation 24 . The local vortex density was obtained by inverting the cell area, and is shown in Fig. 5 (bottom) for every vortex versus its coordinate $x$. The vortex density near the wall clearly exceeds the background density of $\Phi_{0} N_{0} \approx 0.3 \mathrm{mT}$. The theoretical curve $N_{0}+\delta N(x)$, plotted in Fig. 5 (bottom), was calculated using $\varphi=20^{\circ}$ determined from Fig. 1, and a wall width of $2 W=0.6 \mu \mathrm{m}$. The calculated curve reproduces very well not only the sign, but also the magnitude of the excess vortex density. This agreement was achieved using the penetration depth $\lambda=200 \mathrm{~nm}$ as an adjustable parameter. This value of $\lambda$ corresponds to the temperature of $6.8 \mathrm{~K}$ (slightly below $T_{c}=7.2 \mathrm{~K}$ ) which is thus the temperature when the vortices got frozen. The vortex length $L=160 \mu \mathrm{m}$ was another free parameter. It is smaller than the crystal thickness $300 \mu \mathrm{m}$ to compensate for the overestimation of the Abrikosov interaction term in the continuum approximation.

An open question remains regarding the large apparent width of the Bloch wall, approximately $3 \mu \mathrm{m}$ as seen from the image in Fig. 1. The theoretical es- timate obtained by minimizing the sum of exchange, anisotropy and magnetostatic energies is given implicitly by the equation $14(1+w)^{-2}+\alpha w^{-2}=1-K_{u} / \mu_{0} M_{s}^{2}$, where $w=2 W / h$ is the normalized wall width and $\alpha=2 \pi^{2} A / \mu_{0} M_{s}^{2} h^{2}$. Substituting the effective exchange constant $A=2 \times 10^{-11} \mathrm{~J} / \mathrm{m}$ and the uniaxial anisotropy constant $K_{u} \sim 10^{3} \mathrm{~J} / \mathrm{m}^{3}[14]$ we obtain $2 W \approx 0.6 \mu \mathrm{m}$. The discrepancy between the observed and estimated wall width is probably due to the optical diffraction which significantly distorts the image of objects of the order of the light wavelength, $0.55 \mu \mathrm{m}$.

In conclusion, mobile domain walls found in in-plane magnetized ferrite garnet films were investigated for possible use as magnetic micro-manipulators. It was shown, choosing superconducting vortices as a case example, that such films can serve to both apply forces and simultaneously monitor the results of the action. A theoretical model for the interaction was developed, with the vortices described within the London approximation, and the domain wall represented by a charged magnetic wall. The charged wall model, which includes magnetic poles on all the sides of the wall's rectangular cross-section, is shown to give a very good quantitative description of the attraction of vortices to such a wall. The comparison was made by direct observation of individual vortices using the magneto-optical imaging technique.

This work was supported financially by The Norwegian Research Council, Grant No. 158518/431 (NANOMAT) and by FUNMAT@UIO. We gratefully acknowledge discussions with V. Vlasko-Vlasov, L. Uspenskaya, and E. Il'yashenko.
1 W. Hänsel et al., Nature 413, 498 (2001).

${ }^{2}$ H. Ott et al., Phys. Rev. Lett. 87, 230401 (2001).

3 S. Aubin et al., Nature Physics 2, 384 (2006).

4 T. R. Strick et al., Science 271, 1835 (1996).

${ }^{5}$ C. Gosse and V. Croquette, Biophysical Journal 82, 3314 (2002).

${ }^{6}$ R. Folman et al., Phys. Rev. Lett. 84, 4749 (2000).

7 C. Haber and D. Wirtz, Rev. Sci. Instr. 71, 4561 (2000).

8 A. Shevchenko et al., Phys. Rev. A 73, 051401(R) (2006).

9 L. E. Helseth, Langmuir 21, 7276 (2005).

10 L. E. Helseth, T. M. Fischer, and T. H. Johansen, Phys. Rev. Lett. 91, 208302 (2003).

11 P. E. Goa et al., Appl. Phys. Lett. 82, 79 (2003).

12 Ch. Jooss et al., Rep. Prog. Phys. 65, 651 (2002).

13 C. S. Oglesby et al., J. Cryst. Growth 137, 289-294 (1994).

14 L. E. Helseth et al., Phys. Rev. B 65, 132514 (2002).

15 M. V. Milosevic, S. V. Yampolskii, and F. M. Peeters,
Phys. Rev. B 66, 174519 (2002).

16 I. S. Burmistrov and N. M. Chtchelkatchev, Phys. Rev. B 72, 144520 (2005).

17 L. E. Helseth, Phys. Rev. B 66, 104508 (2002).

18 V. K. Vlasko-Vlasov, L. M. Dedukh, and V. I. Nikitenko, Sov. Phys. JETP 44, 1208 (1976).

19 A. Hubert and R. Schäfer, Magnetic Domains (Springer, 1998).

20 G. Carneiro and E. H. Brandt, Phys. Rev. B 61, 6370 (2000).

21 Z. Yang et al., Nature Materials 3, 793-798 (2004).

22 J. Pearl, J. Appl. Phys. 37, 4139 (1966).

${ }^{23}$ P. E. Goa et al., Rev. Sci. Instrum. 74, 141 (2003).

24 C. B. Barber, D. P. Dobkin, and H. Huhdanpaa, ACM TOMS. 22, 469 (1996). 